\title{
On Subsequence Sums of a Zero-sum Free Sequence
}

\author{
Fang Sun \\ Center for Combinatorics, LPMC \\ Nankai University, Tianjin, P.R. China \\ sunf ang2005@163.com
}

Submitted: Jan 16, 2007; Accepted: Jul 18, 2007; Published: Jul 26, 2007

Mathematics Subject Classification: 11B

\begin{abstract}
Let $G$ be a finite abelian group with exponent $m$, and let $S$ be a sequence of elements in $G$. Let $f(S)$ denote the number of elements in $G$ which can be expressed as the sum over a nonempty subsequence of $S$. In this paper, we show that, if $|S|=m$ and $S$ contains no nonempty subsequence with zero sum, then $f(S) \geq 2 m-1$. This answers an open question formulated by Gao and Leader. They proved the same result with the restriction $(m, 6)=1$.
\end{abstract}

\section{Introduction}

Let $G$ be a finite abelian group of order $n$ and exponent $m$, additively written. Let $S=\left(a_{1}, \ldots, a_{k}\right)$ be a sequence of elements in $G$. By $\sum(S)$ we denote the set that consists of all elements of $G$ that can be expressed as the sum over a nonempty subsequence of $S$, i.e.,

$$
\sum(S)=\left\{a_{i_{1}}+\ldots+a_{i_{l}}: 1 \leq i_{1}<\ldots<i_{l} \leq k\right\}
$$

We write $f(S)=\left|\sum(S)\right|$. If $0 \notin \sum(S)$, we call $S$ a zero-sum free sequence.

Let $\sum_{n}(S)$ denote the set that consists of all elements in $G$ which can be expressed as the sum over a subsequence of $S$ of length $n$, i.e.,

$$
\sum_{n}(S)=\left\{a_{i_{1}}+\ldots+a_{i_{n}}: 1 \leq i_{1}<\ldots<i_{n} \leq k\right\}
$$

If $U$ is a subsequence of $S$, we write $S U^{-1}$ for the subsequence obtained by deleting the terms of $U$ from $S$; if $U$ and $V$ are disjoint subsequences of $S$, we write $U V$ for the subsequence obtained by adjoining the terms of $U$ to $V$; if $U$ is a subsequence of $\mathrm{S}$, we write $U \mid S$. 
Let $D(G)$ be the Davenport's constant of $G$, i.e., the smallest integer $d$ such that every sequence $S$ of elements in $G$ with $|S| \geq d$ satisfies $0 \in \sum(S)$; let $s(G)$ be the smallest integer $t$ such that every sequence of elements in $G$ with $|S| \geq t$ satisfies $0 \in \sum_{n}(S)$. In 1961, Erdős, Ginzburg and Ziv proved $s(G) \leq 2 n-1$ for any finite abelian group of order $n$. This result is now well known as the Erdős-Ginzburg-Ziv theorem. In 1996, Gao proved $s(G)=D(G)+n-1$ for any finite abelian group of order $n$. In 1999, Bollobás and Leader investigated the problem of determining the minimal cardinality of $\left|\sum_{n}(S)\right|$ in terms of the length of $|S|$ assuming that $0 \notin \sum_{n}(S)$.

For every positive integer $r$ in the interval $\{1, \ldots, D(G)-1\}$, where $D(G)$ is the Davenport constant of $G$, let

$$
f_{G}(r)=\min _{S,|S|=r}\left|\sum(S)\right|
$$

where $S$ runs over all zero-sum free sequences of $r$ elements in $G$.

In 2006, Gao and Leader proved the following result:

Theorem A.[8] Let $\mathrm{S}$ be a sequence of elements in a finite abelian group of order $n$. Suppose $|S| \geq n$ and $0 \notin \sum_{n}(S)$. Set $r=|S|-n+1$. Then, $\left|\sum_{n}(S)\right| \geq f_{G}(r)$. The equality can be achieved when we take $S=(\underbrace{0, \ldots, 0}_{n-1}, a_{1}, \ldots, a_{r})$, where $\left(a_{1}, \ldots, a_{r}\right)$ is a zero-sum free sequence in $G$ with $f\left(\left(a_{1}, \ldots, a_{r}\right)\right)=f_{G}(r)$.

If $1 \leq r<m$, it is easy to see that $f_{G}(r)=r$, where $m$ is the exponent of $G$. However, when $r \geq m$, the problem of determining $f_{G}(r)$ becomes difficult. Gao and Leader[8] proved $f_{G}(m)=2 m-1$ with the restriction $(m, 6)=1$. They also conjectured the same result without the restriction $(m, 6)=1$. In this paper we show that $f_{G}(m)=2 m-1$ still holds without that restriction.

Theorem 1. If $G$ is a finite non-cyclic abelian group of exponent $m$, then $f_{G}(m)=2 m-1$.

Corollary 1 Let $G$ be a finite abelian group of order $n$ and exponent $m$, and let $S$ be a sequence of elements in $G$ with $|S|=n+m-1$. Then, either $0 \in \sum_{n}(S)$ or $\left|\sum_{n}(S)\right| \geq 2 m-1$.

Proof. It follows from Theorem A and Theorem 1 immediately.

\section{Proof of Theorem 1}

Lemma 2. [2] Let $G$ be an abelian group, and let $S$ be a zero-sum free sequence of elements in $G$. Let $S_{1}, \ldots, S_{t}$ be disjoint nonempty subsequences of $S$. Then, $f(S) \geq \sum_{i=1}^{t} f\left(S_{i}\right)$.

Lemma 3. [3] Let $S$ be a zero-sum free sequence consisting of three distinct elements in an abelian group $G$. If no element in $S$ has order 2 , then $f(S) \geq 6$. 
Lemma 4. Let $S$ be a zero-sum free sequence in $G$. If there is some element $g$ in $S$ with order two, then $\left|\sum(S)\right| \geq 2|S|-1$.

Proof. Set $k=|S|$. Suppose $S=\left(g, a_{1}, \ldots, a_{k-1}\right)$. Since $S$ is zero-sum free and $g=-g$, we have that

$$
\begin{aligned}
& a_{1}, a_{1}+a_{2}, \ldots, a_{1}+a_{2}+\ldots+a_{k-1} \\
& g, g+a_{1}, g+a_{1}+a_{2}, \ldots, g+a_{1}+a_{2}+\ldots+a_{k-1}
\end{aligned}
$$

are $2 k-1$ pairwise distinct elements in $\sum(S)$. Therefore,

$$
\left|\sum(S)\right| \geq 2 k-1
$$

Lemma 5. Let $S=S_{1} S_{2}$ be a zero-sum free sequence in $G$. Let $H=\left\langle S_{1}\right\rangle$ be the subgroup of $G$ generated by $S_{1}$. Let $\phi$ be the natural homomorphism from $G$ onto $G / H$. Set $h=\left|\phi\left(\{0\} \bigcup \sum\left(S_{2}\right)\right)\right|=\left|\left(\{0\} \bigcup \sum\left(S_{2}\right)\right)+H / H\right|$. Then

$$
f(S) \geq h f\left(S_{1}\right)+h-1 .
$$

Proof. Set $A=\{0\} \cup \sum\left(S_{1}\right)$. Since $S$ is zero-sum free, we infer that $0 \notin \sum\left(S_{1}\right)$. Therefore,

$$
|A|=1+f\left(S_{1}\right)
$$

Suppose

$$
\phi\left(\{0\} \bigcup \sum\left(S_{2}\right)\right)=\left\{\phi\left(a_{0}\right), \phi\left(a_{1}\right), \ldots, \phi\left(a_{h-1}\right)\right\},
$$

where $a_{0}=0$ and $a_{i} \in \sum\left(S_{2}\right)$ for $i=1, \ldots, h-1$. Since $A \subseteq H=\left\langle S_{1}\right\rangle$, we infer that

$$
A \backslash\{0\}, a_{1}+A, \ldots, a_{h-1}+A
$$

are pairwise disjoint subsets of $\sum(S)$. Therefore

$$
\begin{aligned}
f(S) & \geq|A \backslash\{0\}|+\left|a_{1}+A\right|+\ldots+\left|a_{h-1}+A\right| \\
& =h f\left(S_{1}\right)+h-1 .
\end{aligned}
$$

For every $a \in G$, write $v_{a}(S)$ for the number of occurrences of $a$ in $S$.

Lemma 6. Let $S$ be a zero-sum free sequence in $G$. Choose $g \in G$ so that $v_{g}(S)=$ $\max _{a \in S}\left\{v_{a}(S)\right\}$. Then $f(S) \geq 2|S|-1$ or $v_{g}(S) \geq \frac{4|S|-f(S)}{6}$. 
Proof. By Lemma 4 we may assume that $S$ contains no element with order 2.

Let $l \geq 0$ be the maximal integer $t$ such that $S$ contains $t$ disjoint subsets each consisting of three distinct elements. Let $A_{1}, \ldots, A_{l}$ be $l$ disjoint 3-subsets of $S$ such that the residual sequence $T=S\left(A_{1} \ldots A_{l}\right)^{-1}$ contains as many distinct elements as possible. Clearly, $T$ can be written in the form

$$
T=(\underbrace{a, \ldots, a}_{u}, \underbrace{b, \ldots, b}_{v})
$$

where $u \geq v \geq 0$ and $u+v=|T|$.

We distinguish two cases:

Case 1. $u \leq 1$. If $v=0$, then $l=\frac{|S|-u}{3}$. Since $S$ contains no element with order 2 , by Lemma 2 and Lemma 3 ,

$$
\begin{aligned}
f(S) & \geq \sum_{i=1}^{l} f\left(A_{i}\right)+|T| \\
& \geq 6 l+u \\
& =2|S|-u \\
& \geq 2|S|-1 .
\end{aligned}
$$

Now assume that $v=1$. Then $u=v=1$ and $l=\frac{|S|-2}{3}$. Again by Lemmas 2 and 3 ,

$$
\begin{aligned}
f(S) & \geq \sum_{i=1}^{l} f\left(A_{i}\right)+f((a, b)) \\
& \geq 6 l+3 \\
& =2|S|-1 .
\end{aligned}
$$

Case 2. $u \geq 2$. If $a \notin A_{i}$ for some $1 \leq i \leq l$, take $c \in A_{i}$ with $c \neq b$ and set $A_{i}^{\prime}=\left(A_{i} \backslash\{c\}\right) \cup\{a\}$. Then $A_{1}, \ldots, A_{i-1}, A_{i}^{\prime}, A_{i+1}, \ldots, A_{l}$ are $l$ disjoint 3 -subsets of $S$ and the residual sequence contains one more distinct elements than $T$ does, a contradiction to the choice of $A_{1}, \ldots, A_{l}$. This shows that $a \in A_{i}$ for every $i \in\{1, \ldots, l\}$. Therefore

$$
v_{g}(S) \geq l+u
$$

By Lemma 2 and Lemma 3, we have that

$$
\begin{aligned}
f(S) & \geq \sum_{i=1}^{l} f\left(A_{i}\right)+v f(a, b)+(u-v) f(a) \\
& \geq 6 l+3 v+u-v \\
& =6 l+u+2 v .
\end{aligned}
$$


Hence

$$
6 l+u+v \leq f(S)-v .
$$

Combining $3 l+u+v=|S|$ with (1), we obtain that

$$
3(2 l+u+v) \geq 4|S|-f(S)+v \geq 4|S|-f(S) .
$$

Therefore,

$$
v_{g}(S) \geq l+u \geq \frac{2 l+u+v}{2}=\frac{3(2 l+u+v)}{6} \geq \frac{4|S|-f(S)}{6} .
$$

Lemma 7. [12] Let $G=C_{n_{1}} \oplus C_{n_{2}}$ with $n_{1} \mid n_{2}$. Then $D\left(C_{n_{1}} \oplus C_{n_{2}}\right)=n_{1}+n_{2}-1$.

Lemma 8. [12] Every sequence $S$ in $C_{n} \bigoplus C_{n}$ with $|S|=3 n-2$ contains a zero-sum subsequence $T$ with $1 \leq|T| \leq n$.

Proof of Theorem 1. Let $S=\left(a_{1}, \ldots, a_{m}\right)$ be a zero-sum free sequence of $m$ elements in $G$. We have to prove that $f(S) \geq 2 m-1$. Choose $g \in G$ so that $v_{g}(S)=\max _{a \in S}\left\{v_{a}(S)\right\}$. By Lemma 6, we may assume that

$$
v_{g}(S) \geq \frac{4|S|-f(S)}{6} \geq \frac{4 m-(2 m-2)}{6}=\frac{m+1}{3},
$$

else the proof is complete.

Let $H$ be the cyclic subgroup generated by $g$. Write $S=S_{1} S_{2}$ such that all terms of $S_{1}$ are in $H$ and no term of $S_{2}$ is in $H$. Hence $\left\langle S_{1}\right\rangle=\langle g\rangle=H$ and $\left|S_{1}\right| \geq v_{g}(S) \geq \frac{m+1}{3}$. Let $\phi$ be the projection from $G$ to $G / H$. Let

$$
S_{2}=\left(b_{1}, \ldots, b_{w}\right),
$$

and set

$$
\phi\left(S_{2}\right)=\left(\phi\left(b_{1}\right), \ldots, \phi\left(b_{w}\right)\right) .
$$

If there is a subsequence $W$ of $S_{2}$ with $|W| \leq 3$ such that $\mid\{0\} \bigcup \sum(\phi(W) \mid) \geq 4$, then by Lemma 2 and Lemma 5, we have that

$$
\begin{aligned}
f(S) & \geq f\left(S_{1} W\right)+f\left(S_{2} W^{-1}\right) \\
& \geq 4 f\left(S_{1}\right)+3+f\left(S_{2} W^{-1}\right) \\
& \geq 4 f\left(S_{1}\right)+3+\left|S_{2}\right|-|W| \\
& \geq 4\left|S_{1}\right|+3+\left|S_{2}\right|-|W| \\
& \geq 4\left|S_{1}\right|+\left|S_{2}\right| \\
& =3\left|S_{1}\right|+m>2 m-1 .
\end{aligned}
$$


Therefore, we may assume that

$$
\left|\{0\} \bigcup \sum \phi(W)\right| \leq 3
$$

for every subsequence $W$ of $S_{2}$ with $|W| \leq 3$.

Let us fix $a \in S_{2}$. For every $b \in S_{2}$, since $\left|\sum(\phi(a), \phi(b)) \bigcup\{0\}\right| \leq 3$, we infer that $\phi(a)=\phi(b)$, or $\phi(a) \neq \phi(b)$ and $\phi(a)+\phi(b)=0$. Therefore,

$$
S_{2}=\left(a+k_{1} g, \ldots, a+k_{u} g,-a+l_{1} g, \ldots,-a+l_{v} g\right),
$$

where $u \geq v \geq 0$ and $u \geq 1$ and $k_{i}, l_{j} \in\{0,1, \ldots, m-1\}$.

Let $G_{0}=\langle a, g\rangle$ be the subgroup of $G$ generated by $a$ and $g$. Clearly, $\left|G_{0}\right|=$ $|\langle\phi(a)\rangle||\langle g\rangle|=\operatorname{ord}(\phi(a)) \operatorname{ord}(g)$. Observe that $S$ is a zero-sum free sequence in $\langle S\rangle=G_{0}$. We distinguish two cases:

Case 1: $\operatorname{ord}(\phi(a))=2$, i.e., $2 a \in\langle g\rangle=H$. Since $S$ is zero-sum free we have $v_{g}(S)<\operatorname{ord}(g)$. Therefore, $\operatorname{ord}(g)>\frac{m+1}{3}$. Hence $\operatorname{ord}(g)=m$ or $\operatorname{ord}(g)=\frac{m}{2}$. If $\operatorname{ord}(g)=\frac{m}{2}$, then $\left|G_{0}\right|=m$ and $D\left(G_{0}\right) \leq m=|S|$, a contradiction to the fact that $S$ is zero-sum free. Therefore, $\operatorname{ord}(g)=m$ and

$$
G_{0} \cong C_{2} \bigoplus C_{m}
$$

By Lemma 7 , it follows that $D\left(G_{0}\right)=m+1$.

For an arbitrary $g^{\prime} \in G_{0} \backslash\{0\}$, set $T=S\left(-g^{\prime}\right)$. Then $|T|=m+1=D\left(G_{0}\right)$. Therefore, $T$ contains a nonempty zero-sum subsequence $W$. Since $S$ is zero-sum free, $W=W_{0}\left(-g^{\prime}\right)$ with $W_{0} \mid S$. Therefore, $\sigma\left(W_{0}\right)+\left(-g^{\prime}\right)=0$, or $g^{\prime}=\sigma\left(W_{0}\right) \in \sum(S)$. This shows that $\sum(S)=G_{0} \backslash\{0\}$. Therefore,

$$
f(S)=\left|\sum(S)\right|=\left|G_{0}\right|-1=2 m-1 .
$$

Case 2: $\operatorname{ord}(\phi(a)) \geq 3$. Hence $m \geq 3$. If $u=1$ and $v=0$, then by Lemma 5 it follows that

$$
f(S) \geq 2 f\left(S_{1}\right)+1 \geq 2\left|S_{1}\right|+1=2 m-1 .
$$

If $u=2$ and $v=0$, then since $\operatorname{ord}(\phi(a)) \geq 3$, it follows that

$$
\left|\sum\left(\phi\left(a+k_{1} g\right), \phi\left(a+k_{2} g\right)\right) \cup\{0\}\right|=3 .
$$

Hence, since $m \geq 3$, it follows in view of Lemma 5 that

$$
f(S) \geq 3 f\left(S_{1}\right)+2 \geq 3\left|S_{1}\right|+2=3(m-2)+2 \geq 2 m-1 .
$$


Now assume that either $u \geq 3$, or else $u=2$ and $v \geq 1$. Hence, if $\operatorname{ord}(\phi(a)) \geq 4$, then either

$$
\left|\{0\} \cup \sum\left(\phi\left(a+k_{1} g\right), \phi\left(a+k_{2} g\right), \phi\left(a+k_{3} g\right)\right)\right| \geq 4,
$$

or

$$
\left|\{0\} \cup \sum\left(\phi\left(a+k_{1} g\right), \phi\left(a+k_{2} g\right), \phi\left(-a+l_{1} g\right)\right)\right| \geq 4,
$$

contradicting inequality (2) in both cases. Therefore, we conclude that

$$
\operatorname{ord}(\phi(a))=3
$$

Hence,

$$
\left|G_{0}\right|=3(\operatorname{ord}(g)) \quad \text { and } 3 \mid m \text {. }
$$

From the proof of Case 1, we know that $\operatorname{ord}(g)=m$ or $\operatorname{ord}(g)=\frac{m}{2}$. If $\operatorname{ord}(g)=\frac{m}{2}$, then $\left|G_{0}\right|=\frac{3 m}{2}$. It follows from $\exp \left(G_{0}\right) \mid m$ that $G_{0}=C_{3} \bigoplus C_{\frac{m}{2}}$. Hence by Lemma 7 , it follows that $D\left(G_{0}\right)=\frac{m}{2}+2 \leq m=|S|$, a contradiction. Hence $\operatorname{ord}(g)=m$ and

$$
G_{0}=C_{3} \bigoplus C_{m}
$$

From $\operatorname{ord}(\phi(a))=3$, we infer that $3 a=k g$ for some $k \geq 0$. Therefore, $\frac{m}{3} k g=m a=0$. Hence, $m \mid \frac{m}{3} k$. This gives that $3 \mid k$. Set $q=\frac{k}{3}$. Thus $3 a=3 q g$. Set $a^{\prime}=a-q g$. Hence $3 a^{\prime}=0$ and $\operatorname{ord}\left(\phi\left(a^{\prime}\right)\right)=3$. Clearly,

$$
S_{2}=\left(a^{\prime}+k_{1}^{\prime} g, \ldots, a^{\prime}+k_{u}^{\prime} g, 2 a^{\prime}+l_{1}^{\prime} g, \ldots, 2 a^{\prime}+l_{v}^{\prime} g\right),
$$

where $k_{i}^{\prime}=k_{i}+q$ and $l_{j}^{\prime}=l_{j}-q$.

Now we have that

$$
G_{0}=\left\langle a^{\prime}\right\rangle \oplus\langle g\rangle .
$$

Let $H_{0}=\left\langle a^{\prime}\right\rangle \bigoplus\left\langle\frac{m}{3} g\right\rangle$. Note $H_{0} \cong C_{3} \bigoplus C_{3}$. Let $\rho$ be the homomorphism from $G_{0}$ onto $H_{0}$ defined by :

$$
\rho\left(r a^{\prime}+s g\right)=r a^{\prime}+\frac{m}{3} s g
$$

Clearly, $\operatorname{ker}(\rho)=\langle 3 g\rangle \cong C_{\frac{m}{3}}$.

Since $v_{g}(S) \geq \frac{m+1}{3}$ and $m \geq 3$, it follows that $v_{g}(S) \geq 2$. Set $S_{0}=S\left(a^{\prime}+k_{1}^{\prime} g, a^{\prime}+\right.$ $\left.k_{2}^{\prime} g, g, g\right)^{-1}$. Hence,

$$
S=\left(a^{\prime}+k_{1}^{\prime} g, a^{\prime}+k_{2}^{\prime} g\right)(g, g) S_{0} .
$$

Suppose $m \geq 9$. Hence applying Lemma 8 to the sequence $\rho\left(S_{0}\right)$ in $H_{0} \cong C_{3} \bigoplus C_{3}$, one can find $\frac{m}{3}-3$ disjoint subsequences $T_{1}, \ldots, T_{\frac{m}{3}-3}$ of $S_{0}$ such that

$$
\sigma\left(\rho\left(T_{i}\right)\right)=0 \text { and } 1 \leq\left|T_{i}\right| \leq 3
$$


The residual sequence $S_{0}\left(T_{1} \ldots T_{\frac{m}{3}-3}\right)^{-1}$ has length

$$
\begin{aligned}
\left|S_{0}\left(T_{1} \ldots T_{\frac{m}{3}-3}\right)^{-1}\right| & =\left|S_{0}\right|-\left|T_{1} \ldots T_{\frac{m}{3}-3}\right| \\
& \geq m-4-3\left(\frac{m}{3}-3\right) \\
& =5 \\
& =D\left(C_{3} \oplus C_{3}\right)=D\left(H_{0}\right) .
\end{aligned}
$$

Therefore, $S_{0}\left(T_{1} \ldots T_{\frac{m}{3}-3}\right)^{-1}$ contains a nonempty subsequence $T_{\frac{m}{3}-2}$ (say) such that $\sigma\left(\rho\left(T_{\frac{m}{3}}-2\right)\right)=0$. Now we have

$$
\sigma\left(T_{i}\right) \in \operatorname{ker}(\rho)=\langle 3 g\rangle \cong C_{\frac{m}{3}}
$$

for every $i \in\left\{1,2, \ldots, \frac{m}{3}-2\right\}$.

Since $S$ is zero-sum free, we know that $\left(a+k_{1}^{\prime} g, a+k_{2}^{\prime} g, g, g, \sigma\left(T_{1}\right), \ldots, \sigma\left(T_{\frac{m}{3}-2}\right)\right)$ is also zero-sum free. By Lemma 5 and Lemma 2, we have that

$$
\begin{aligned}
f\left((g, g)\left(\sigma\left(T_{1}\right), \ldots, \sigma\left(T_{\frac{m}{3}-2}\right)\right)\right) & \geq 3 f\left(\sigma\left(T_{1}\right), \ldots, \sigma\left(T_{\frac{m}{3}-2}\right)\right)+2 \\
& \geq 3\left(\frac{m}{3}-2\right)+2 \\
& =m-4 .
\end{aligned}
$$

Again, by Lemma 5 and Lemma 2, we have that

$$
\begin{aligned}
& f\left(\left(a+k_{1}^{\prime} g, a+k_{2}^{\prime} g, g, g, \sigma\left(T_{1}\right), \ldots, \sigma\left(T_{\frac{m}{3}-2}\right)\right)\right) \\
& \geq 3 f\left((g, g)\left(\sigma\left(T_{1}\right), \ldots, \sigma\left(T_{\frac{m}{3}-2}\right)\right)\right)+2 \\
& \geq 3(m-4)+2 \\
& =3 m-10 .
\end{aligned}
$$

Since $m \geq 9$, it follows that $f(S) \geq 3 m-10 \geq 2 m-1$.

So, we may assume that $m \leq 8$. Consequently, since $3 \mid m$, it follows that $m=3$ or $m=6$. Note that $v_{g}(S) \geq \frac{m+1}{3}$ and $u \geq 2$. Therefore, $\frac{m+1}{3}+2 \leq|S|=m$. Hence $m>3$. Thus, $m=6$.

Since $v_{g}(S) \geq \frac{m+1}{3}$, we have that $\left|S_{1}\right| \geq 3$. Thus by Lemma 5 ,

$$
\begin{aligned}
f(S) & \geq f\left(S_{1}\left(a^{\prime}+k_{1}^{\prime} g, a^{\prime}+k_{2}^{\prime} g\right)\right) \\
& \geq 3 f\left(S_{1}\right)+2 \\
& \geq 3\left|S_{1}\right|+2 \\
& \geq 3 \cdot 3+2=2 \cdot 6-1 .
\end{aligned}
$$

This proves that $f(S) \geq 2 m-1$. 
The following example shows that $f_{G}(m)=2 m-1$. Let $a, b$ be elements in $G$ with $\operatorname{ord}(a)=m$ and $b \notin\langle a\rangle$. Let $S=(\underbrace{a, \ldots, a}_{m-1}, b)$. Clearly, $S$ is zero-sum free and $f(S)=$ $2 m-1$. This completes the proof.

Acknowledgments. I thank the referee and Professor W.D. Gao for their helpful suggestion and comments. This work was supported by the 973 Project, the PCSIRT Project of the Ministry of Education, the Ministry of Science and Technology, and the National Science Foundation of China.

\section{References}

[1] B. Bollobás, I. Leader, The number of k-sums modulo $k$, J. Number Theory 78 (1999), 27-35.

[2] J.D. Bovey, P. Erdős, I. Niven, Conditions for zero sum modulo n, Canda. Math. Bull. 18 (1975), 27-29.

[3] R.B. Eggleton, P. Erdős, Two combinatorial problems in group theroy, Acta Arith. 21 (1972), 111-116.

[4] P. Erdős, A. Ginzburg, A. Ziv, A theorem in additive number theory, Bull. Res. Council Israel 10F (1961), 41-43.

[5] W.D. Gao, Addion thorems for finite abelian groups, J. Number Theory 53 (1995), 241-246.

[6] W.D. Gao, A combinatorial problem on finite abelian groups, J. Number Theory 58 (1996), 100-103.

[7] W.D. Gao, A. Geroldinger, On the structure of zero-sum-free sequences, Combinatorica 18 (1998), 519-527.

[8] W.D. Gao, I. Leader, Sums and k-sums in abelian groups of order $k$, J. Number Theory 1 (2006), 1-7.

[9] A. Gerolinger, R. Schneider, On Davenport's constant, J. Combin. Theory Ser. A 61 (1992), 147-152.

[10] Y.O. Hamidoune, O. Ordaz, A. Ortuńo, On a combinatorial theorem of Erdös, Ginzburg and Ziv, Combin. Probab. Comput. 7 (1998), 403-412.

[11] J.E. Olson, A combinatorial problem on finite abelian groups I, J. Number Theory 1 (1969), 8-10.

[12] J.E. Olson, A combinatorial problem on finite abelian groups II, J. Number Theory 1 (1969), 195-199.

[13] J.E. Olson, An addition theorem for finite abelian groups, J. Number Theory 9 (1977), 63-70. 